\title{
Sensitive real-time $P C R$ detection of Plasmodium falciparum parasites in whole blood by erythrocyte membrane protein 1 gene amplification
}

\author{
Bryan Grabias ${ }^{1}$, Edward Essuman ${ }^{1}$, Isabella A. Quakyi ${ }^{2}$ and Sanjai Kumar ${ }^{1,3^{*}}$
}

\begin{abstract}
Background: Malaria remains a global public health problem responsible for 445,000 deaths in 2016. While microscopy remains the mainstay of malaria diagnosis, highly sensitive molecular methods for detection of low-grade sub-microscopic infections are needed for surveillance studies and identifying asymptomatic reservoirs of malaria transmission.

Methods: The Plasmodium falciparum genome sequence was analysed to identify high copy number genes that improve P. falciparum parasite detection in blood by RT-PCR. Plasmodium falciparum erythrocyte membrane protein 1 (PfEMP1)-specific primers were evaluated for P. falciparum detection in hospital-based microscopically positive dried blood spots and field-acquired whole blood from asymptomatic individuals from Ghana.

Results: PfEMP1 outperformed the Pf18S sequence for amplification-based P. falciparum detection. PfEMP1 primers exhibited sevenfold higher sensitivity compared to Pf18S primers for parasite genomic DNA. Probit analysis established a 95\% detection threshold of 9.3 parasites $/ \mathrm{mL}$ for PfEMP1 compared to 98.2 parasites $/ \mathrm{mL}$ for Pf1 $8 \mathrm{~S}$ primers. The PfEMP1 primers also demonstrated superior clinical sensitivity, identifying 100\% (20/20) of dried blood spot samples and 70\% (69/98) of asymptomatic individuals as positive versus 55\% (11/20) and 54\% (53/98), respectively, for Pf18S amplification.
\end{abstract}

Conclusions: These results establish PFEMP1 as a novel amplification target for highly sensitive detection of both acute infections from filter paper samples and submicroscopic asymptomatic low-grade infections.

Keywords: Plasmodium falciparum erythrocyte membrane protein 1, Parasitemia, Real-time PCR, Genomic DNA

\section{Background}

In 2016, malaria caused approximately 445,000 deaths, primarily among young children in sub-Saharan Africa [1]. The apicomplexan Plasmodium parasites responsible for malaria-related illness are transmitted through the bite of infected mosquitoes. Of the five recognized species that infect human beings (Plasmodium

\footnotetext{
*Correspondence: Sanjai.Kumar@fda.hhs.gov

${ }^{1}$ Laboratory of Emerging Pathogens, Division of Emerging

and Transfusion Transmitted Diseases, Center for Biologics Evaluation and Research, Food and Drug Administration, Silver Spring, MD 20993, USA

Full list of author information is available at the end of the article
}

falciparum, Plasmodium vivax, Plasmodium malariae, Plasmodium ovale, and Plasmodium knowlesi), P. falciparum is the most common and the most lethal. Malaria also presents a public health challenge in the USA. While there have been no cases of autochthonous transmission of malaria in the USA since 1957 [2], increasing globalization and travel to areas where malaria is endemic still results in imported malaria cases in the Plasmodium. In 2015 alone, there were 1186 of such travel-related malaria cases reported to the Center for Disease Control (CDC) [3] and the number of malaria hospitalizations from 2000 to 2014 far exceeded that of other common travel-related illnesses 
[4]. Additionally, malaria is also a major risk to the blood supply in both endemic and non-endemic countries [5-7].

The gold standard of malaria parasite detection is direct visual confirmation of intraerythrocytic parasites in a blood film. However, this method is time and labourintensive and can only reach typical detection limits of 5000-150,000 parasites/mL of blood [8]. Several reports utilizing PCR-based methods to detect $P$. falciparum in blood have achieved far greater sensitivities than standard blood smears $[9,10]$. Given the lack of knowledge on the minimum number of parasites that could be present in asymptomatic individuals and limitations in volume of blood used in detection assays, molecular methods of improved sensitivity are still needed to identify all Plasmodium infections in endemic areas.

Typically, most nucleic acid-based assays rely upon the detection of pathogen specific 18S ribosomal RNA (rRNA) sequences due to their universal presence, strong inter- and intra-species sequence conservation, and the relatively high abundance. While the given quantity of 18S rRNA transcripts may be higher than the number of gene copies present in an organism's genome, RNA is typically less stable and thus requires more stringent sample handling than genomic DNA (gDNA). Depending on the strain, P. falciparum parasites may possess only 5-8 gene copies of the $18 \mathrm{~S}$ ribosomal subunit [11]; therefore, the performance of assays that amplify and detect genomic sequences can be greatly enhanced with the identification of novel biomarkers that are more abundant than Pf18S sequences. In this report, PCR-based nucleic acid assays utilizing primers specific to the $P$. falciparum $18 \mathrm{~S}$ ribosomal gene sequence and highly conserved regions of the abundant (approximately 60 variants [12]) P. falciparum var. gene EMP1 are developed and characterized. Data from analytical studies demonstrate that $P$. falciparum detection using the PfEMP1 primer set consistently outperforms the traditional $18 \mathrm{~S}$ primer set, detecting as litthe as $0.01 \mathrm{pg}$ of Plasmodium gDNA and 9.3 parasites $/ \mathrm{mL}$ compared to $0.07 \mathrm{pg}$ and 98.2 parasites $/ \mathrm{mL}$ for the $P f 18 \mathrm{~S}$ primer set. The clinical performance of the PfEMP1 and Pf18S gene targets was determined in hospital-based blood film positive clinical malaria samples collected on filter paper and in field acquired blood samples of asymptomatic individuals from Ghana. PfEMP1 amplification consistently outperforms $P f 18 \mathrm{~S}$-based RT-PCR detection, identifying $100 \%$ (20/20) of dried blood spot samples and 70\% (69/98) of asymptomatic individuals as positive versus $55 \%(11 / 20)$ and $54 \%(53 / 98)$, respectively, for $18 \mathrm{~S}$ amplification. In conclusion, a PfEMP1-based RTPCR test has applications in detecting acute filter paper collected and low-grade asymptomatic infections in field who have a potential to convert into severe malaria in children and serve as reservoirs of transmission in both low and high transmission endemic areas.

\section{Methods \\ Primers}

Primer oligonucleotides were purchased from Eurofin Genomics (Louisville, Kentucky). The primer sequences employed in this study are: 18S F: CTTAACCTGCTA ATTAGCG, 18S R: ATTCCTCGTTCAAGA TTAATA ATT; EMP1 F: AAGAAAACGAATTATTTGGGACA, EMP1 R: AGAAACATCAGTATTCAACGTT. SYBR green RT-PCR mastermix was purchased from Bio-Rad (Hercules, CA, USA).

\section{Human blood samples}

Plasmodium falciparum-infected blood smear positive samples from patients in Ghana used for this study were collected on Whatman ${ }^{\circledR}$ FTA Elute cards (GE Lifesciences, MA, USA) at Kpone Katamanso District Hospital in the Greater Accra Region of Ghana, a malaria endemic country which recorded about 10 million malaria cases in the year 2015. The study site was developed for multidisciplinary malaria studies by the University of Ghana School of Public Health [13]. Kpone Katamanso district is a peri-urban district located in the coastal-savannah zone that occupies an area of $215.4 \mathrm{~km}^{2}$ and has an estimated population of 50,225 . While malaria transmission intensity of the region varies seasonally, infections are acquired year-round. Study participants were 1 month to 16 years old children and asymptomatic adults who were screened in the Kpone Katamanso District Hospital.

\section{Preparation of Plasmodium falciparum gDNA}

Plasmodium falciparum 3D7 parasites were cultured in vitro according to previously published protocols [14] utilizing $\mathrm{O}^{+}$human red blood cells purchased from Interstate Blood Bank (Memphis, TN). Parasitaemia was determined by routine Giemsa staining of blood smears. Final parasitaemia was measured and the number of parasitized red blood cells (pRBCs) per $\mu \mathrm{L}$ was obtained. Isolated ring stage P. falciparum parasites from culture were spiked into whole blood and serially diluted. For spiked sample analysis, gDNA was extracted from $1 \mathrm{~mL}$ of whole blood at each parasite dilution. Alternately, approximately $100-200 \mu \mathrm{L}$ of blood was utilized from capillary collection tubes collected from asymptomatic blood smear negative adults from Ghana. For both spiked and Ghanaian blood samples, lysis of RBCs was induced by adding saponin (Sigma, MO, USA) to a final concentration of $0.15 \%$. Remaining cellular material was sedimented by centrifugation at $16,000 \times g$ for $10 \mathrm{~min}$ and washed three times with $1 \times$ PBS. gDNA from the pellet was obtained using the QIAmp DNA Blood Mini Kit 
Fig. 1 Abundance of PfEMP1-specific amplicon in Plasmodium falciparum genome. Primers used in the RT-PCR assay amplify a region of 260 nucleotides located in the cytosolic acidic terminal segment (ATS) region of PfEMP1. Matching conserved sequences in the P. falciparum (3D7) genome were identified using an NCBI BLAST search. Chromosomes with homologous sequences are displayed in the figure. The conserved amplification region was found in 18 different loci reflecting significantly higher abundance than the 5 copies of the 185 gene in the P. falciparum genome. Black bars represent forward and reverse primer binding positions

according to the manufacturer's instructions and eluted in $60 \mu \mathrm{L}$ ultrapure water. gDNA extracts from dried blood samples spotted onto Whatman ${ }^{\circledR}$ FTA Elute cards (were prepared according to the manufacturer's instructions.

\section{Real-time polymerase chain reaction}

Real-time amplification reactions were performed on a QuantStudio 3 RT-PCR instrument (Applied Biosystems, Foster City, CA, USA) using a SYBR Green mastermix from Biorad (CA, USA). The thermocycler protocol was: $95^{\circ} \mathrm{C}$ for $5 \mathrm{~min}$; a 40 repeat cycle of $95^{\circ} \mathrm{C}$ for $15 \mathrm{~s}, 57^{\circ} \mathrm{C}$ for $30 \mathrm{~s}$, and data collection and elongation at $72{ }^{\circ} \mathrm{C}$ for $1 \mathrm{~m}$. Cut-off threshold cycle values were determined for each RT-PCR plate run based upon the mean threshold of included negative (uninfected) control well results minus 1 standard deviation. All results are displayed as signal-to-cut-off ratios using a simple $\Delta C_{t}$ method (Fold $=2^{\text {(Mean Ct,cutoff }- \text { Mean Ct,sample) }}$.

\section{Probit analysis}

Approximately $40 \mathrm{~mL}$ of whole blood was spiked with differing concentrations of $P$. falciparum ring stage parasites (ranging from 1 to 100 parasites $/ \mathrm{mL}$ ). Genomic DNA was isolated from 30 unique $1 \mathrm{~mL}$ aliquots drawn from these larger volumes. Extracted genomic DNA from each aliquot was tested by real-time PCR amplification utilizing both the $P f 18 \mathrm{~S}$ and PfEMP1 primer sets. The MedCalc statistical software package (MedCalc Software, Belgium) was used to perform probit regression on the RT-PCR data and determine the $95 \%$ reactivity threshold and corresponding confidence intervals.

\section{Results}

\section{Sequence analysis of PfEMP1 gene amplicon in $P$. falciparum genome}

To identify a novel and abundant target sequence for RTPCR-based detection of P. falciparum genomic DNA that would potentially outperform standard $18 \mathrm{~S}$ amplification methods, the sequences of members of the PfEMP1 multi-gene family, which consists of approximately 60

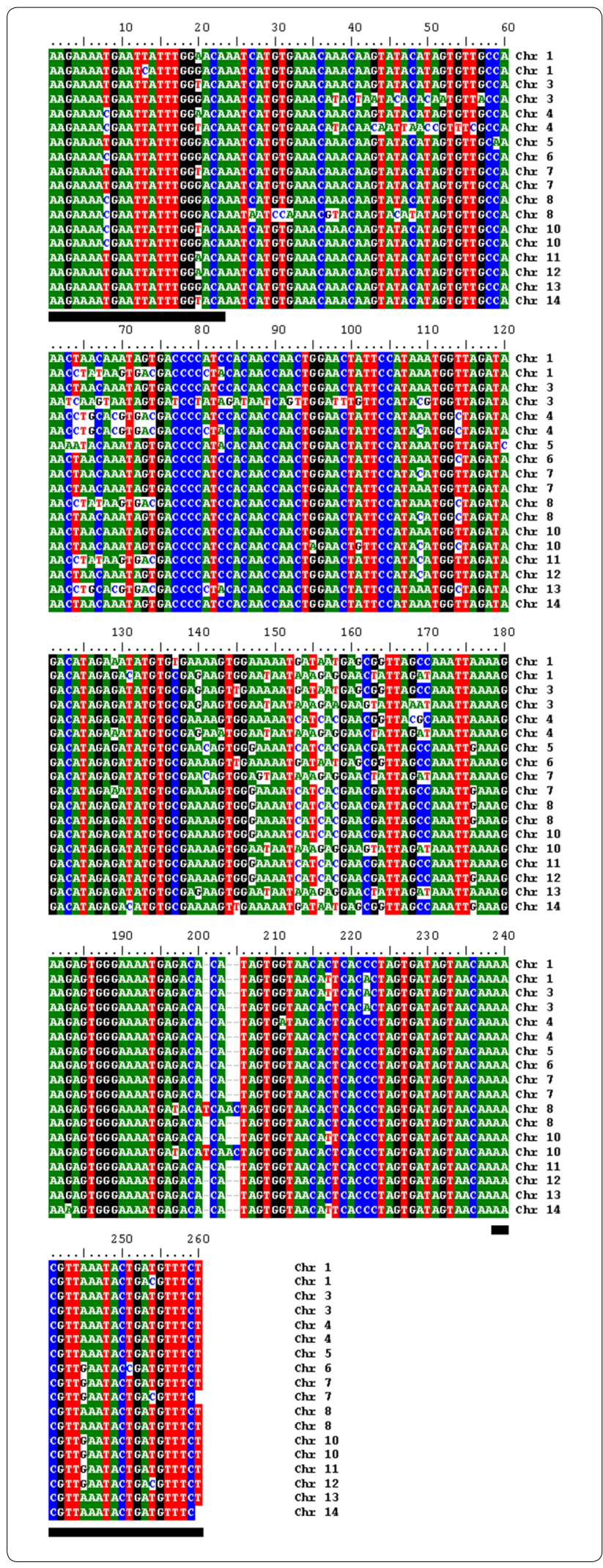


unique variants present per genome, were analysed. Specifically, primers were designed to amplify strongly conserved gene regions that maximize the number of amplification targets. Primer sequences exhibited 91.3-100\% identity to a maximum of 18 possible sites of amplification (Fig. 1) within the cytosolic acidic terminal segment of PfEMP1. As this sequence resides on the intracellular side of the erythrocyte, there is substantially less selective pressure for antigenic variation. A maximum of two base-pair mismatches far from the $3^{\prime}$ end of each primer was allowed. Thus, when compared to the 5-8 available genomic copies of $P f 18 \mathrm{~S}$ ribosomal subunit present, this PfEMP1-based RT-PCR assay would potentially improve detection capability by offering a more abundant amplification target.

\section{Limit of detection of $P$. falciparum gDNA}

To compare the sensitivity of Pf18S and PfEMP1 as detection targets, both primers were evaluated in RTPCR against purified parasite gDNA that was serially diluted in ultrapure water. The $P f 18 \mathrm{~S}$ primer set detected as few as $0.07 \mathrm{pg}$ of $P f$ gDNA (Fig. 2a) whereas the PfEMP1 primer set detected as little as $0.01 \mathrm{pg}$ of $P f$ gDNA (Fig. 2b) showing a sevenfold improved sensitivity in detection of $P$. falciparum blood stage parasite gDNA.

\section{Determination of primer limit of detection (LOD) by probit analysis}

Approximately $40 \mathrm{~mL}$ of whole blood was spiked with either $100,10,5$, or 1 parasite/mL of P. falciparum bloodstage parasite. gDNA was extracted from 30 unique $1 \mathrm{~mL}$ aliquots taken from each stock $40 \mathrm{~mL}$ preparation and evaluated in RT-PCR using both Pf18S and PfEMP1 primer sets. The MedCalc statistical software package was used to perform probit regression analysis to calculate the parasite concentration at the $95 \%$ detection threshold. The probit analysis demonstrates that the $95 \%$ detection limit is 98.2 parasites/mL (95\% CI
64.5-131.9 parasites/mL) for the $P f 18 \mathrm{~S}$ primers (Fig. 3a) and 9.3 parasites $/ \mathrm{mL}$ (95\% CI $6.36-17.8$ parasites $/ \mathrm{mL}$ ) for the $P f$ EMP1 primer set (Fig. 3b).

\section{Detection of $P$. falciparum in dried blood spots and serum samples from Ghana}

To evaluate the clinical sensitivity of each primer set, Ghanaian blood samples that were microscopically positive were assessed for $P$. falciparum reactivity by RTPCR. Dried blood spots (DBS) employ a small drop of blood on filter paper and are highly utilized in endemic areas due to their relative ease of use and stability at room temperature [15]. Briefly, patient specimens were collected on Whatman FTA-elute filter paper cards in volumes of approximately $10-20 \mu \mathrm{L}$ and gDNA was isolated according to the manufacturer's instructions. Importantly, the sensitivity of any pathogen detection assay utilizing dried blood spots is generally lower than whole blood due to the relatively small volume being sampled $[16,17]$. To empirically estimate the difference in analytical sensitivity of the RT-PCR assay between gDNA extracted from dried blood spots versus whole blood, gDNA was extracted from both $20 \mu \mathrm{L}$ of parasite-spiked blood and $20 \mu \mathrm{L}$ of unspiked, uninfected blood utilized as a negative control that were spotted onto FTA elute cards (Fig. 4a). The Pf18S primer set only marginally detected the highest parasite concentration tested $(41,360$ parasites $/ \mathrm{mL})$ while the PfEMP1 primer set successfully reacted to as few as 2640 parasites $/ \mathrm{mL}$. The superior sensitivity of PfEMP1 amplification was also confirmed by analysis of dried blood spots collected from individuals in a malaria endemic area of Ghana. The Pf $18 \mathrm{~S}$ primer set identified only $11 / 20$ (55\%) samples as positive, with $9 / 20$ samples showing no amplification at all (Fig. 4b). In contrast, the PfEMP1 primer set successfully identified 20/20 (100\%) blood smear positive samples as positive (Fig. 4b).
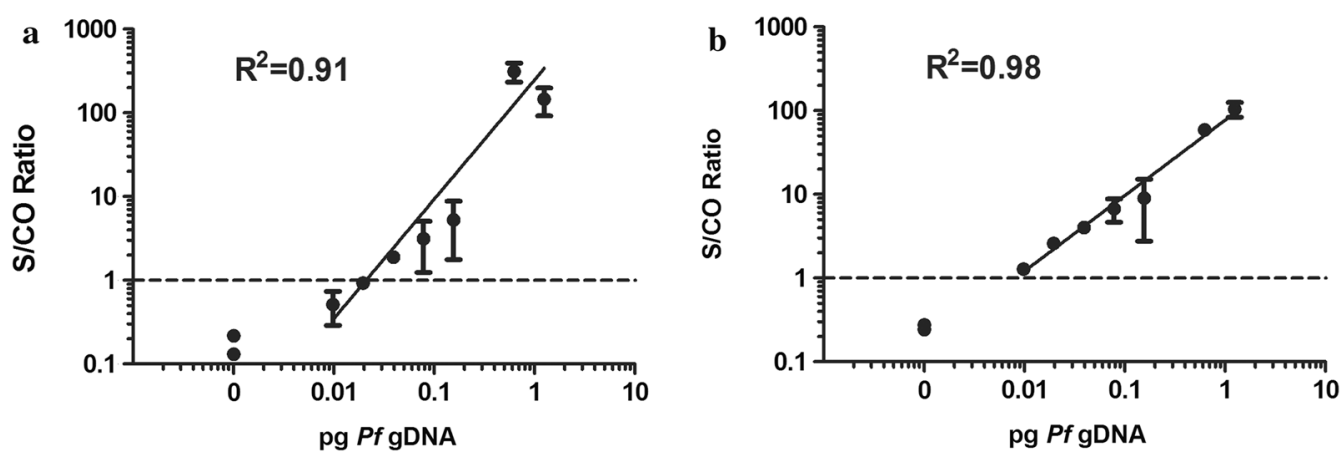

Fig. 2 Analytical sensitivity of Pf18S and PfEMP1 primers for serially diluted parasite genomic DNA (gDNA). A linear regression identifying the limit of detection for each primer set at the cutoff threshold of the assay. a The 185 primers detected as little as $0.07 \mathrm{pg}$. b PfEMP1 primers could detect 0.01 pg of parasite gDNA 

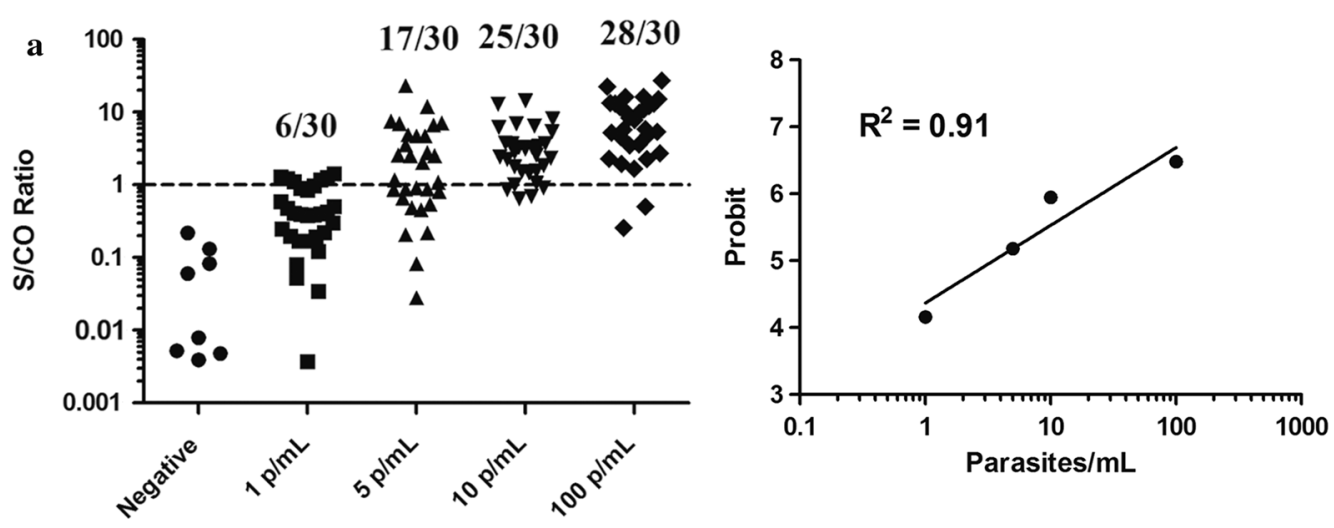

Pf Parasite Concentration

$30 / 30$
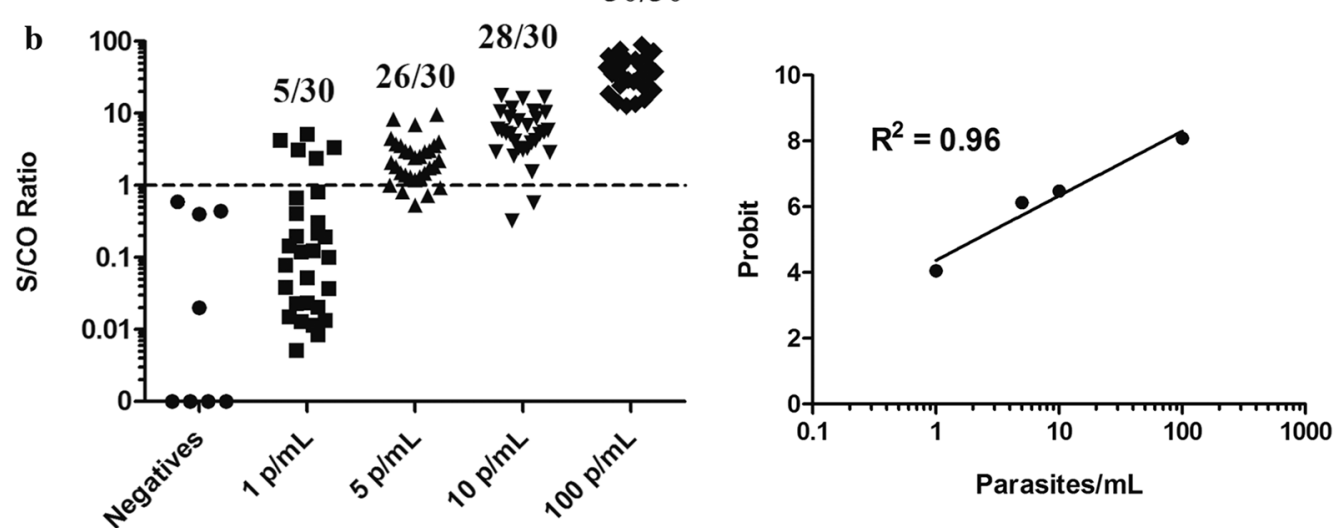

Pf Parasite Concentration

Fig. 3 Determination of analytical sensitivity for Plasmodium falciparum-infected red blood cells by probit analysis. gDNA extracted from 30 independent $1 \mathrm{~mL}$ aliquots taken from the stock parasite dilutions and analysed by RT-PCR using both primer sets. Probit regression was performed using the MedCalc statistical software to identify the $95 \%$ reactive threshold. a The $18 \mathrm{~S}$ primer set demonstrated a $95 \%$ reactivity of 98.2 parasites/ $\mathrm{mL}$ (95\% Cl 64.5-131.9). b PfEMP1 primer set demonstrated a 95\% threshold of 9.3 parasites $/ \mathrm{mL}$ (95\% Cl 6.36-17.8)

Performance of both Pf18S and PfEMP1 primer sets for P. falciparum detection in serum was also evaluated. Patient serum, as opposed to whole blood samples which are typically assessed in parasite nucleic acid tests, are more commonly stored long-term [18]. Thus, adequate detection of $P$. falciparum from stored serum samples could allow for more extensive retrospective analyses on existing patient-acquired material. These results demonstrated that, while both primer sets showed no amplification in the healthy adult samples, only the EMP1 primer set successfully identified $5 / 7(71.4 \%)$ sera from infected individuals as positive (Fig. 4c, d).

\section{Detection of $P$. falciparum in asymptomatic blood smear negative whole blood samples}

The clinical performance of $P f 18 \mathrm{~S}$ and $P f E M P 1$ primers in detecting submicroscopic asymptomatic infections was evaluated in individuals living in an endemic area of Ghana. Specifically, these blood samples reflect asymptomatic adults who were diagnosed as negative for $P$. falciparum infection by blood film microscopy. Pf18S primers identified 53/98 (54.1\%) of samples as positive for $P$. falciparum gDNA whereas PfEMP1 primers successfully detected 69/98 (70.4\%) (Fig. 5, Table 1). These differences in sensitivity are statistically significant as measured via McNemar's test $(\mathrm{P}<0.01)$.

\section{Discussion}

Blood-film microscopy is considered the gold-standard for malaria diagnosis. While generally adequate for diagnosis of acute malaria, microscopy lacks the sensitivity to detect low-grade asymptomatic infections as revealed by the application of molecular tests that detect the parasite nucleic acid. Since submicroscopic malaria infections are important sources of further transmission, identification of this population group is critical for accurate estimation 


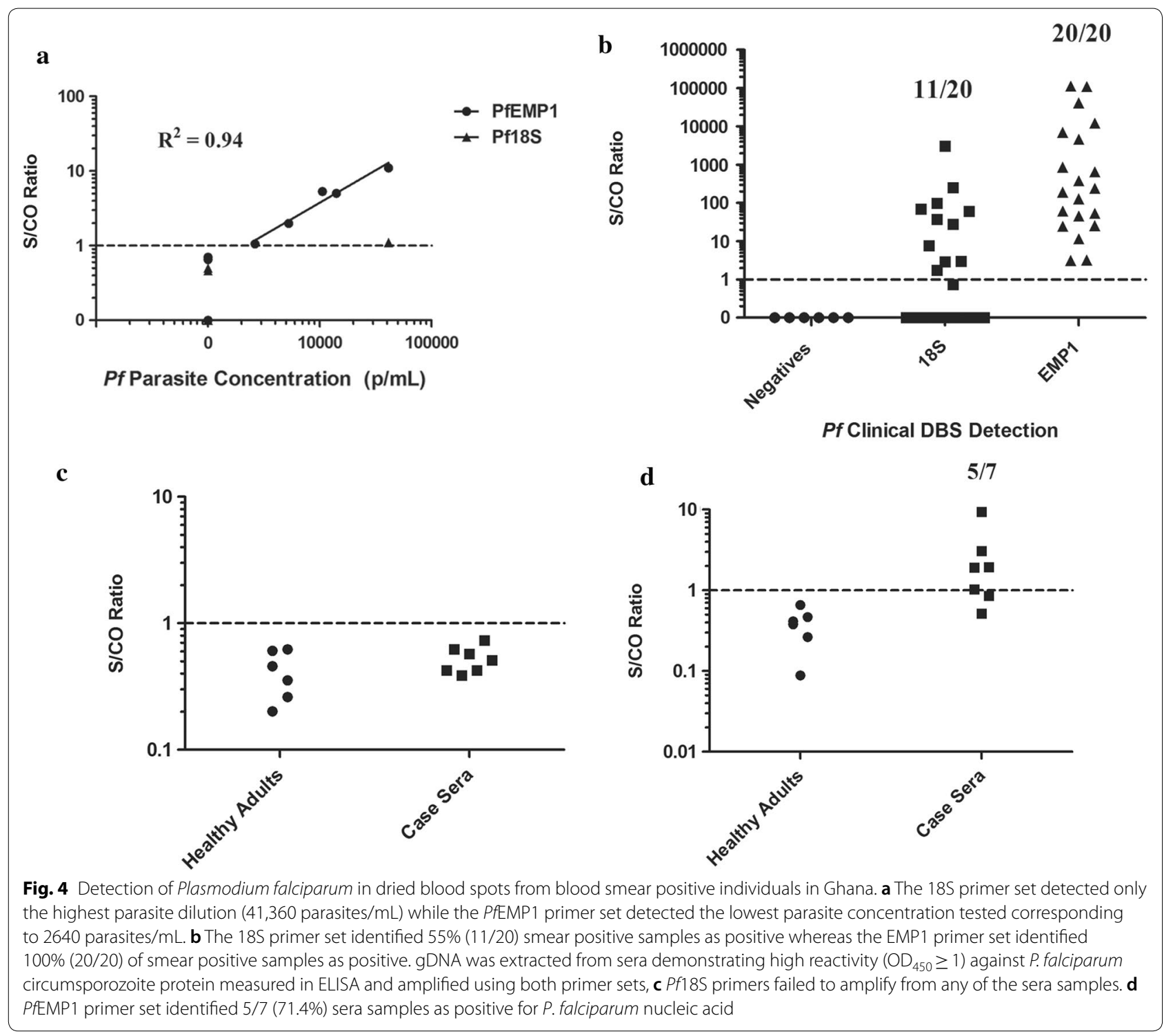

of malaria incidence rate in an endemic area. In semiimmune individuals who live with chronic exposure to malaria parasites, asymptomatic low-grade parasitaemia can persist for a year or more [19]. Failure to comprehensively identify these individuals infected with low burden parasitaemias that may not be detected by currently used microscopy, PCR assays, or Rapid Diagnostic Tests, dramatically reduces the effectiveness of Mass Screen and Treat (MSAT) strategies targeting the infectious reservoir of malaria [20,21].

In this work, the performance of an RT-PCR-based screening assay for $P$. falciparum employing primers that amplify members of the PfEMP1 var gene family was characterized. The data establish that PfEMP1 primers exhibit a superior analytical sensitivity of 9.3 parasites/ $\mathrm{mL}$ in whole blood versus the 98.2 parasites $/ \mathrm{mL}$ limit of detection for the standard Pf $18 \mathrm{~S}$ amplification. Accordingly, amplification of PfEMP1 identified 16\% more whole blood samples from asymptomatic adults who were blood film negative as positive than standard $P f 18 \mathrm{~S}$ amplification establishing the superior efficacy of this molecule in detecting low grade parasite burdens missed by other methods.

Results from analytical and clinical characterization studies suggest that the PfEMP1-based RT-PCR assay could provide a more comprehensive estimation of the infectious reservoir of malaria and simultaneously allow better targeting of treatment and transmission-blocking interventions. In particular, this assay would reduce falsenegative results associated with malaria screening assays of inadequate sensitivity in areas of less intense transmission where chronic parasite burdens are generally 


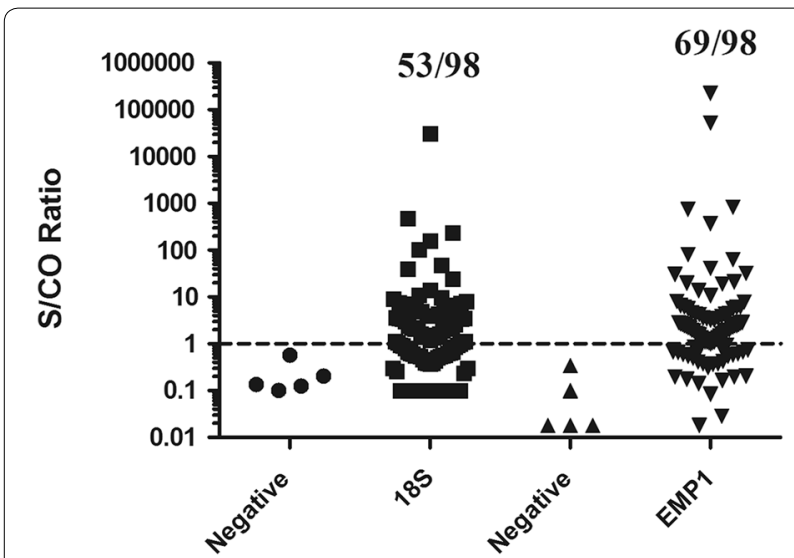

Fig. 5 Detection of Plasmodium falciparum in asymptomatic blood smear negative individuals from Ghana. gDNA was extracted from whole blood samples collected from children and adults who exhibited no symptoms of malaria and had negative blood smears. Pf18S primers identified 53/98 (54.1\%) of samples as positive for P. falciparum gDNA whereas PfEMP1 primers identified 69/98 (70.4\%) blood samples as positive. These differences are statistically significant when evaluated by McNemar's test and demonstrate PfEMP1 primers possess superior sensitivity for the detection of low burden parasitaemia and more comprehensive identification of infectious reservoir

Table 1 Detection of Plasmodium falciparum gDNA in asymptomatic individuals from Ghana

\begin{tabular}{cccc}
\hline & EMP1 & Total \\
\cline { 2 - 3 } & + & - & \\
\hline 185 & & 9 & \\
+ & 44 & 20 & 53 \\
- & 25 & 29 & 45 \\
Total & 69 & & 98 \\
\hline
\end{tabular}

low [22]. Additionally, modeling studies have shown that dramatic increases in the sensitivity of malaria diagnostic assays, like that observed with PfEMP1-based amplification, are required to exert any substantial effect on malaria transmission in regions of high intensity. According to one report, application of higher sensitivity assays accelerates the interruption of malaria transmission in an area. Accordingly, deployment of diagnostic assays of superior sensitivity would render overall public health benefits and reduce the organizational and overhead costs of running programmes, such as MSAT campaigns thus remaining a critical component of global malaria eradication efforts [21].

Collection of blood samples as DBS on filter paper is highly amenable for field studies due to ease of sample collection and storage. However, detection of $P$. falciparum from the smaller volumes of blood typically collected as DBS is less sensitive than screening larger volumes of whole blood [16, 17]. Additionally, a recent study has shown that overall PCR reactivity of even high parasite burden DBS declines over time when not stored at $-20{ }^{\circ} \mathrm{C}$ [23]. These fundamental sample constraints necessitate a more sensitive diagnostic assay to ensure no loss of parasite detection in valuable field samples acquired in epidemiological or vaccine-related studies. The results demonstrate that PfEMP1-based RT-PCR amplification improved $P$. falciparum parasite detection of microscopically positive Ghanaian blood samples as DBS when compared to standard Pf18S amplification methods. Specifically, PfEMP1-mediated gene amplification demonstrated a sensitivity of $2460 \mathrm{p} / \mathrm{mL}$ versus a limit of detection of at least 41,360 parasites $/ \mathrm{mL}$ for $18 \mathrm{~S}$-based detection. Additionally, PfEMP1 primers successfully amplified $100 \%$ of blood smear positive individuals from dried blood spot samples whereas $P f 18 \mathrm{~S}$ amplification only detected 11/20 (55\%) DBS samples as positive. Importantly, the failure of $P f 18 \mathrm{~S}$ amplification to consistently match results from gold standard blood smear microscopy of clinical samples collected as DBS indicates a limitation in this blood collection method and inherent challenges in conducting large field studies in endemic areas. Cumulatively, these data demonstrate that RT-PCR amplification of PfEMP1 provides a superior method of parasite detection among DBS spots collected in field sites and ensures that any potential losses of sensitivity are minimized when this widely used sample format is employed.

The analytical and clinical performance studies establish PfEMP1 as a superior biomarker for RT-PCR-based detection of both submicroscopic infections in samples collected as whole blood and patent parasitaemias from samples collected as DBS. These results support the identification of potentially novel abundant diagnostic markers as a means of improving the sensitivity of pathogen gDNA detection. While PfEMP1 is a well- known and characterized molecule associated with malaria pathogenesis [24-26], no reports have thus far attempted to harness this high copy multi-gene family as an amplification target in nucleic acid-based screening assays for malaria. All PfEMP1 variants are high-molecular weight parasite proteins $(200-450 \mathrm{kDa})$ inserted into the membranes of $P$. falciparum-infected red blood cells. PfEMP1 is the major constituent protein of knobs that are responsible for sequestration of infected RBCs in the peripheral vasculature that allows immune evasion of later-stage parasites [27, 28]. The conserved cytosolic portion of PfEMP1 is anchored to the RBC cytoskeleton by lysine-rich membrane associated PHISTb (LyMP) $[29,30]$. Although 60 variants of PfEMP1 exist, only a single PfEMP1 is expressed during any given 48 -h cycle. 
All other unexpressed versions of parasite PfEMP1 are silenced by multiple different mechanisms such as histone modifications [31], promoter-intron 'pairing' [32], and tethering of the var genes at the nuclear periphery [33]. Thus, while PfEMP1 may not serve as a superior transcript for RNA-based amplification assays, the high number of gene copies of PfEMP1 in the malaria genome makes it an ideal candidate diagnostic marker for the detection of parasite DNA in blood. In this work, primers were designed to amplify a strongly conserved gene sequence encoding the cytosolic acidic terminal segment (ATS) region of PfEMP1. The finalized forward and reverse primers possess at least $91 \%$ identity to 18 different loci within the $P$. falciparum genome which offer more abundant targets of amplification than the available 5-8 genomic copies of the $18 \mathrm{~S}$ ribosomal subunit gene. While the extracellular portion of PfEMP1 is subject to immense selective pressure to evade the immune system, the cytosolic domain amplified in the assay and located in the second exon of the gene is significantly less diverse $[34,35]$.

\section{Conclusion}

Apart from developing countries, malaria also presents a challenge in industrialized countries where autochthonous transmission does not occur. In these countries, malaria presents a significant public health challenge through imported clinical cases and as transfusion transmitted malaria [4, 5]. Global malaria surveillance is a priority of the World Health Organization, however, reported cases are often incomplete, lacking confirmatory testing, and many cases are unreported because of misdiagnosis. Most incidences of imported or transfusion-related cases in nonendemic countries can be traced to prior residents of malaria endemic areas [36]. Due to acquired immunity, these individuals would be potentially asymptomatic and maintain an infectious reservoir characterized by low parasite burden. Assays of higher sensitivity would be required to identify prospective at-risk blood donors who may have travelled to or were prior residents of malaria endemic areas. In summary, this work established an RT-PCR-based assay for the detection of parasite gDNA that relies on amplification of PfEMP1 gene sequences. The use of PfEMP1 as a diagnostic marker of malaria infection has demonstrated superior analytical and clinical sensitivity when compared to the current laboratory standard of $18 \mathrm{~S}$ RTPCR amplification; therefore, the proposed assay ensures the detection of even asymptomatic, low parasite burden individuals that present the greatest risk to the blood supply.

\section{Authors' contributions}

$\mathrm{BG}$, analysed data and wrote manuscript. EE collected the samples and performed the experiments. IAQ contributed with clinical samples and study site information. SK conceptualized, designed the study and wrote the manuscript. All authors read and approved the final manuscript.

\section{Author details \\ ${ }^{1}$ Laboratory of Emerging Pathogens, Division of Emerging and Transfusion Transmitted Diseases, Center for Biologics Evaluation and Research, Food and Drug Administration, Silver Spring, MD 20993, USA. ${ }^{2}$ Department of Bio- logical Environmental and Occupational Health Sciences, School of Public Health, College of Health Sciences, University of Ghana, Legon, Ghana. ${ }^{3}$ Laboratory of Emerging Pathogens, Division of Emerging and Transfusion Transmitted Diseases, Center for Biologics Evaluation and Research, Food and Drug Administration, 10903 New Hampshire Ave. Building 52-72 Rm 5304, Silver Spring, MD 20993, USA.}

\section{Acknowledgements}

We are grateful to all the Ghanaian individuals whose blood samples were used in this study. We also thank the technicians for their assistance in processing the blood samples during sera collection.

\section{Competing interests}

The authors declare that they have no competing interests.

\section{Availability of data and materials}

The datasets used and/or analysed during the current study are available from the corresponding author per reasonable request.

\section{Consent for publication}

There are no details on any individuals contained in this publication.

\section{Ethics approval and consent to participate}

This study protocol was approved by the Ghana Health Service ethical review committee (GHS-ERC 06/05/16) and the FDA Research Involving Human Subjects Committee (protocol \#09-122B). All clinical samples used in this study were anonymized.

\section{Funding}

This work was funded by the intramural FDA grant and the Path malaria vaccine initiative.

\section{Publisher's Note}

Springer Nature remains neutral with regard to jurisdictional claims in published maps and institutional affiliations.

Received: 28 August 2018 Accepted: 21 March 2019

Published online: 02 April 2019

\section{References}

1. WHO. World malaria report. Geneva: World Health Organization; 2017

2. Zucker JR. Changing patterns of autochthonous malaria transmission in the United States: a review of recent outbreaks. Emerg Infect Dis. 1996;2:37-43.

3. CDC. Malaria surveillance-United States, 2011. Surveill Summ. 2013;62(ss05):1-17.

4. Khuu D, Eberhard ML, Bristow BN, Javanbakht M, Ash LR, Shafir SC, et al. Malaria-related hospitalizations in the United States, 2000-2014. Am J Trop Med Hyg. 2017;97:213-21.

5. Verra F, Angheben A, Martello E, Giorli G, Perandin F, Bisoffi Z. A systematic review of transfusion-transmitted malaria in non-endemic areas. Malar J. 2018;17:36.

6. Owusu-Ofori A, Gadzo D, Bates I. Transfusion-transmitted malaria: donor prevalence of parasitaemia and a survey of healthcare workers knowledge and practices in a district hospital in Ghana. Malar J. 2016;15:234. 
7. Maier CL, Gross PJ, Dean CL, Chonat S, Ip A, McLemore M, et al. Transfusion-transmitted malaria masquerading as sickle cell crisis with multisystem organ failure. Transfusion. 2018;58:1550-4.

8. Thellier M, Datry A, Alfa Cisse O, San C, Biligui S, Silvie O, et al. Diagnosis of malaria using thick bloodsmears: definition and evaluation of a faster protocol with improved readability. Ann Trop Med Parasitol. 2002;96:115-24.

9. Hofmann N, Mwingira F, Shekalaghe S, Robinson LJ, Mueller I, Felger I. Ultra-sensitive detection of Plasmodium falciparum by amplification of multi-copy subtelomeric targets. PLoS Med. 2015:12:e1001788.

10. Mahajan B, Zheng H, Pham PT, Sedegah MY, Majam VF, Akolkar N, et al. Polymerase chain reaction-based tests for pan-species and speciesspecific detection of human Plasmodium parasites. Transfusion. 2012:52:1949-56.

11. Mercereau-Puijalon O, Barale JC, Bischoff E. Three multigene families in Plasmodium parasites: facts and questions. Int J Parasitol. 2002:32:1323-44.

12. Gardner MJ, Hall N, Fung E, White O, Berriman M, Hyman RW, et al. Genome sequence of the human malaria parasite Plasmodium falciparum. Nature. 2002;419:498-511.

13. Tchouassi DP, Quakyi IA, Addison EA, Bosompem KM, Wilson MD, Appawu MA, et al. Characterization of malaria transmission by vector populations for improved interventions during the dry season in the Kpone-on-Sea area of coastal Ghana. Parasit Vectors. 2012;5:212.

14. Oakley MS, Gerald N, Anantharaman V, Gao Y, Majam V, Mahajan B, et al. Radiation-induced cellular and molecular alterations in asexual intraerythrocytic Plasmodium falciparum. J Infect Dis. 2013;207:164-74.

15. Pritsch M, Wieser A, Soederstroem V, Poluda D, Eshetu T, Hoelscher $\mathrm{M}$, et al. Stability of gametocyte-specific Pfs 25 -mRNA in dried blood spots on filter paper subjected to different storage conditions. Malar J. 2012;11:138.

16. Tran TM, Aghili A, Li S, Ongoiba A, Kayentao K, Doumbo S, et al. A nested real-time PCR assay for the quantification of Plasmodium falciparum DNA extracted from dried blood spots. Malar J. 2014;13:393.

17. Canier L, Khim N, Kim S, Eam R, Khean C, Loch K, et al. Malaria PCR detection in Cambodian low-transmission settings: dried blood spots versus venous blood samples. Am J Trop Med Hyg. 2015;92:573-7.

18. Bharti AR, Patra KP, Chuquiyauri R, Kosek M, Gilman RH, Llanos-Cuentas $A$, et al. Polymerase chain reaction detection of Plasmodium vivax and Plasmodium falciparum DNA from stored serum samples: implications for retrospective diagnosis of malaria. Am J Trop Med Hyg. 2007;77:444-6.

19. Bretscher MT, Maire N, Chitnis N, Felger I, Owusu-Agyei S, Smith T. The distribution of Plasmodium falciparum infection durations. Epidemics. 2011;3:109-18.

20. Tiono AB, Ouedraogo A, Ogutu B, Diarra A, Coulibaly S, Gansane A, et al. A controlled, parallel, cluster-randomized trial of community-wide screening and treatment of asymptomatic carriers of Plasmodium falciparum in Burkina Faso. Malar J. 2013;12:79.

21. Slater HC, Ross A, Ouedraogo AL, White LJ, Nguon C, Walker PG, et al. Assessing the impact of next-generation rapid diagnostic tests on Plasmodium falciparum malaria elimination strategies. Nature. 2015:528:594-101.
22. Satoguina J, Walther B, Drakeley C, Nwakanma D, Oriero EC, Correa S, et al. Comparison of surveillance methods applied to a situation of low malaria prevalence at rural sites in The Gambia and Guinea Bissau. Malar J. 2009;8:274.

23. Schwartz A, Baidjoe A, Rosenthal PJ, Dorsey G, Bousema T, Greenhouse $B$. The effect of storage and extraction methods on amplification of Plasmodium falciparum DNA from dried blood spots. Am J Trop Med Hyg. 2015;92:922-5

24. Chen Q, Barragan A, Fernandez V, Sundstrom A, Schlichtherle M, Sahlen $A$, et al. Identification of Plasmodium falciparum erythrocyte membrane protein 1 (PfEMP1) as the rosetting ligand of the malaria parasite P. falciparum. J Exp Med. 1998;187:15-23.

25. Subramani R, Quadt K, Jeppesen AE, Hempel C, Petersen JE, Hassenkam T, et al. Plasmodium falciparum-infected erythrocyte knob density is linked to the PfEMP1 variant expressed. mBio. 2015;6:e01456-15.

26. Ganguly AK, Ranjan P, Kumar A, Bhavesh NS. Dynamic association of PfEMP1 and KAHRP in knobs mediates cytoadherence during Plasmodium invasion. Sci Rep. 2015;5:8617.

27. Langreth SG, Reese RT. Antigenicity of the infected-erythrocyte and merozoite surfaces in falciparum malaria. J Exp Med. 1979:150:1241-54.

28. Howard RJ, Barnwell JW, Rock EP, Neequaye J, Ofori-Adjei D, Maloy WL, et al. Two approximately 300 kilodalton Plasmodium falciparum proteins at the surface membrane of infected erythrocytes. Mol Biochem Parasitol. 1988;27:207-23.

29. Oberli A, Slater LM, Cutts E, Brand F, Mundwiler-Pachlatko E, Rusch S, et al. A Plasmodium falciparum PHIST protein binds the virulence factor PfEMP1 and comigrates to knobs on the host cell surface. FASEB J. 2014;28:4420-33.

30. Proellocks NI, Herrmann S, Buckingham DW, Hanssen E, Hodges EK, Elsworth B, et al. A lysine-rich membrane-associated PHISTb protein involved in alteration of the cytoadhesive properties of Plasmodium falciparum-infected red blood cells. FASEB J. 2014;28:3103-13.

31. Tonkin CJ, Carret CK, Duraisingh MT, Voss TS, Ralph SA, Hommel M, et al. Sir2 paralogues cooperate to regulate virulence genes and antigenic variation in Plasmodium falciparum. PLoS Biol. 2009;7:e84.

32. Swamy L, Amulic B, Deitsch KW. Plasmodium falciparum var. gene silencing is determined by cis DNA elements that form stable and heritable interactions. Eukaryot Cell. 2011;10:530-9.

33. Ralph SA, Scheidig-Benatar C, Scherf A. Antigenic variation in Plasmodium falciparum is associated with movement of var loci between subnuclear locations. Proc Natl Acad Sci USA. 2005;102:5414-9.

34. Kraemer SM, Smith JD. Evidence for the importance of genetic structuring to the structural and functional specialization of the Plasmodium falciparum var. gene family. Mol Microbiol. 2003;50:1527-38.

35. Lavstsen T, Salanti A, Jensen AT, Arnot DE, Theander TG. Sub-grouping of Plasmodium falciparum 3D7 var genes based on sequence analysis of coding and non-coding regions. Malar J. 2003;2:27.

36. O'Brien SF, Delage G, Seed CR, Pillonel J, Fabra CC, Davison K, et al. The epidemiology of imported malaria and transfusion policy in 5 nonendemic countries. Transfus Med Rev. 2015;29:162-71.

Ready to submit your research? Choose BMC and benefit from

- fast, convenient online submission

- thorough peer review by experienced researchers in your field

- rapid publication on acceptance

- support for research data, including large and complex data types

- gold Open Access which fosters wider collaboration and increased citations

- maximum visibility for your research: over $100 \mathrm{M}$ website views per year

At BMC, research is always in progress.

Learn more biomedcentral.com/submissions 